in utero (the death of the fetus occurs within few days), while during second trimester a hysterotomy as a first step is advised. This reduces the risk of obstetrical complications (bleeding, rupture of the cervix, diffuse intravascular coagulation) and psychological impact on the patient. Alternatively, before chemoradiotherapy is initiated, feticide can be considered for ethical and psychological reasons.

Conclusion: Radical hysterectomy is the treatment of choice for pregnant patients with early cervical cancer. It affords termination or delivery of the pregnancy at the same time of the treatment is provided. Radical hysterectomy during pregnancy can be safely performed even with the fetus in situ and a subsequent cesarean section.

Poster (C17)

Cervical Cancer

https://doi.org/10.3802/jgo.2021.32.S1.C17

\section{Establishment of a novel cell line from glassy cell carcinoma of the cervix and analysis of genomic landscape}

\author{
Yasunari Ishizuka, ${ }^{1,{ }^{*}}$ Masataka Kudo, ${ }^{1}$ Kanako Hatanaka, ${ }^{2}$ \\ Toraji Amano, ${ }^{3}$ Yutaka Hatanaka, ${ }^{2,3}$, Hidemichi Watari' \\ 'Department of Obstetrics and Gynecology, Hokkaido University, \\ Graduate School of Medicine and Faculty of Medicine, Sapporo, Japan \\ (giants_y_i_203@yahoo.co.jp) \\ ${ }^{2}$ Center for Development of Advanced Diagnostics, Hokkaido University \\ Hospital, Sapporo, Japan \\ ${ }^{3}$ Clinical Research and Medical Innovation Center, Hokkaido University \\ Hospital, Sapporo, Japan
}

Objective: Glassy cell carcinoma (GCC) of the cervix is an extremely rare subtype of cervical cancer with poor prognosis, and the molecular characteristics remain unclear. In this study, we investigated the landscape of genomic alterations of GCC. Methods: We established a novel GCC cell line (HU-6) and analyzed genomic alterations of 160 cancer-related genes (GeneRead Human Comprehensive Cancer Panel, Qiagen) in comparison with the parental tumor and 2 existing GCC cell lines available (TOM-2, HOKUG).

Results: HU-6 was derived from a 31-year-old GCC case, and mimicked the parental tumor both in pathohistological findings and in gene alteration patterns. HPV 18 was detected in HU-6 and TOM-2, while HPV 58 in HOKUG. Common gene alterations in 3 GCC cell lines were detected in 40 loci of 26 cancer-related genes, including alterations of the homologous recombination repair (HRR) pathway (ATM, BRCA2, FANCA, and BRIP1), and the SWI/SNF pathway (PBRM1, EZH2), in addition to single nucleotide variant in Noch1 and TP53, which induces differentiation into squamous epithelium, and a frequent and poor prognosis marker in adenocarcinoma of the cervix, respectively. Only HU-6 had a mutation of KRAS (G12C). Copy number analysis revealed that HU-6 showed amplification of Myc, but HOKUG showed loss of heterozygosity of several genes.

Conclusion: HRR related gene alterations were commonly identified in all three GCC cell lines, suggesting that PARP inhibitors may have antitumor effect for GCC. Moreover, KRAS inhibitors such as AMG510 or ARS1620 may be effective for GCC with KARS mutation (G12C).

Poster (C18)

Cervical Cancer

https://doi.org/10.3802/jgo.2021.32.S1.C18

\section{Evaluation of mobile health applications for cervical cancer in the digital marketplace}

\author{
Jakkapop Kanjak, Naratassapol Likitdee, " \\ Chumnan Kietpeerakool, Amornrat Temtanakitpaisan \\ Khon Kaen University, Khon Kaen, Thailand (polli@kku.ac.th)
}

Objective: To assess the quality of mobile health (mHealth) applications (apps) for cervical cancer using the Mobile App Rating Scale (MARS), APPLICATIONS scoring system, and app rating using specific statements.

Methods: We searched for cervical cancer apps from 2 major mobile operating systems (Google Play Store and Apple iTunes Store) in March 2021. Eligible apps were downloaded and assessed for quality by 2 independent reviewers using multimodal assessment tools.

Results: The overall quality MARS score was $2.61 \pm 0.79$ of 5 . The highest scoring app was "ASCCP Management Guidelines" (3.98). Overall, apps scored highest in the functionality domain, followed by information, engagement, and aesthetics. The mean \pm standard deviation of the APPLICATIONS scoring system was $8.50 \pm 1.71$ of 16 . The highest-rated apps were "ASCCP Management Guidelines," "BSCCP," and "Cervical Cancer Guide." Apps scored highest in the paid subscription and price domain. In contrast, apps scored poorly in the text search, literature used, and subjective presentation domains. Concerning app content, many apps infrequently provide misconceptions about cervical cancer. The apps' rating using specific statements was $7.81 \pm 4.56$ of 16 . Conclusion: Overall, the apps analyzed by the MARS and APPLICATIONS scoring systems demonstrated above-average quality. However, there is a need for improvement in the essential information conveyed by these apps. Moreover, the assessment tools have influenced different app quality rating results, confirming a lack of standardized quality assessment tools for them Health app. 